\title{
The Structure of
}

Homeomorphism and

Diffeomorphism Groups

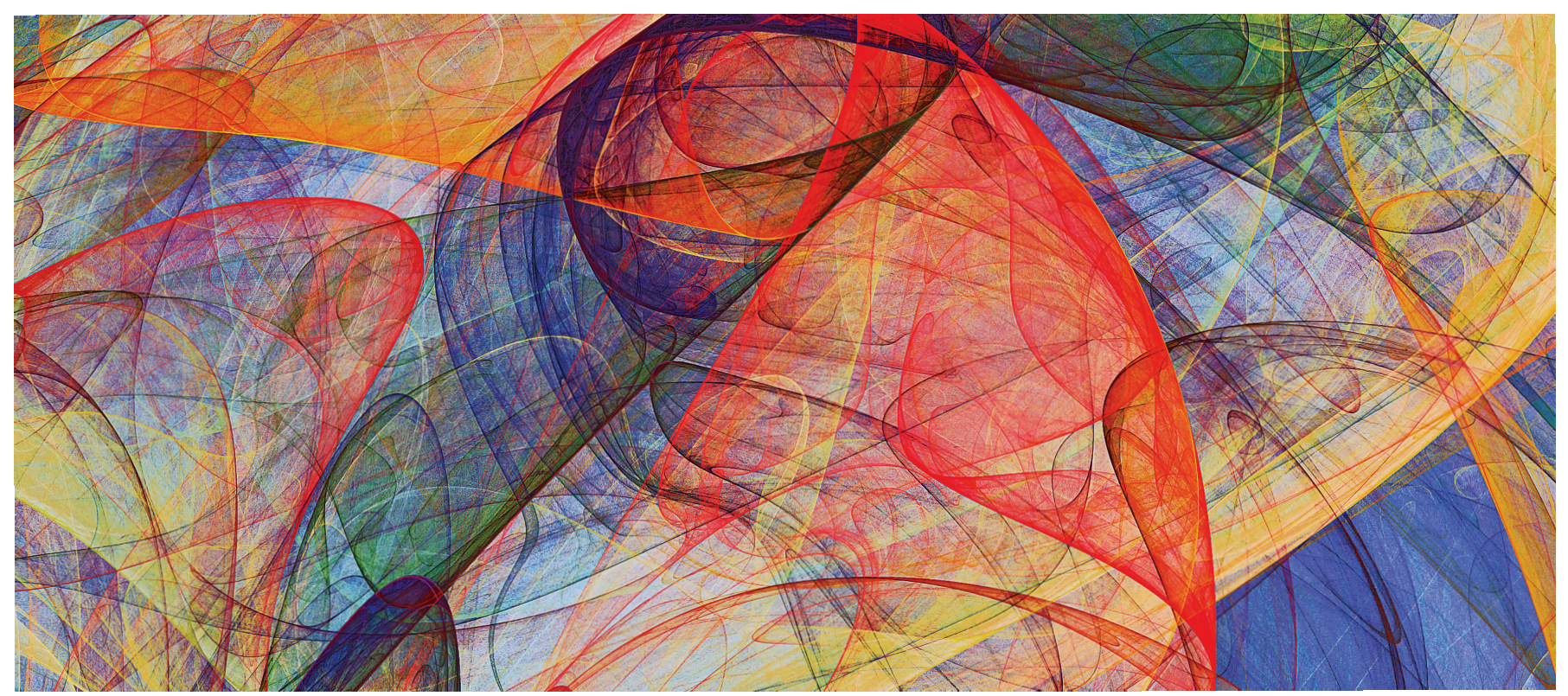

\section{Kathryn Mann}

My favorite opening to a math talk is from a 2014 lecture of Etienne Ghys. Part of a minicourse for young researchers in geometric group theory, he begins the lecture with "My second favorite group [dramatic pause...] is the group of all diffeomorphisms of a compact manifold." Beyond the obvious question what is your first favorite, then? (for this, one should see the rest of the lecture series), this line has another hook that I like even better. Ghys' statement is a bit like answering the question "what is your favorite food" with "my favorite food is dessert!" That's a great response, I couldn't agree more, but didn't you just cheat there by naming an infinite class of things in place of a single thing? For it has been known since the 1980s that varying the manifold $M$ and even varying what you mean by diffeomorphism (smooth, $C^{1}, C^{2}, \ldots$ ) produces an infinite

Kathryn Mann is an assistant professor of mathematics at Cornell University. Her email address is $\mathrm{k}$. mann@corne11. edu.

For permission to reprint this article, please contact:

reprint-permission@ams.org.

DOI: https://doi.org/10.1090/noti2252 family of pairwise nonisomorphic groups. In other words, manifolds are completely classified by the algebraic structure of their diffeomorphism groups.

However, to Ghys' credit, many of the tools we have to approach the study of these groups are broad principles that can be applied to large classes of examples. In fact, several structural results about diffeomorphism groups also hold for groups of homeomorphisms, although the absence of differentiability typically necessitates a different toolkit for the proof. My goal here is to give you a quick tour of what we know of the structure theory of homeomorphism and diffeomorphism groups and highlight a few recent developments both in this theory and in its relationship with the modern study of dynamical systems. To keep this piece within a reasonable scope, I have chosen to focus on the algebraic and dynamical structural properties, leaving absent the equally fascinating world of the topology, especially the homotopy type, of such groups. I recommend Hatcher's A 50-year View of Diffeomorphism Groups [Hat12] as an introduction to what is known, and not known, in this direction. 


\section{Why Study Diffeomorphism Groups?}

We understand mathematical objects by understanding their symmetries-this is the essence of Felix Klein's Erlangen Program and a guiding principle in many areas of mathematics. Following this principle, anyone who studies topological or smooth manifolds should seek to understand the groups $\operatorname{Homeo}(M)$ and $\operatorname{Diff}(M)$ of self-homeomorphisms and, respectively, selfdiffeomorphisms of a manifold $M$. Topologists are also interested in these groups for many other reasons, including the role they play in surgery theory, $K$-theory, and the theory of fiber bundles. To give a concrete and relatively elementary example, the group cohomology of Homeo( $M)$ and $\operatorname{Diff}(M)$ (that is to say the cohomology of their classifying spaces) gives the characteristic classes of topological and smooth $M$-bundles, respectively, while their cohomology as discrete groups gives characteristic classes for flat bundles, or bundles with a foliation transverse to the fibers. Thus, both the homotopy type and the algebraic structure of these groups play a crucial role in classifying fiber bundles over an arbitrary base space.

Another source of motivation for the study of diffeomorphism groups comes from dynamical systems. In its most general sense, dynamics is simply the study of transformations under iteration. Classically, dynamicists studied the behavior of a single transformation and its iterates, that is to say a cyclic subgroup of $\operatorname{Homeo}(M)$ or of $\operatorname{Diff}(M)$. In the past fifty years, the field has widened to encompass the study of larger systems of transformations obeying some algebraic laws, i.e., very general (typically infinite but finitely generated) subgroups of homeomorphism or diffeomorphism groups. A major motivating question is to understand to what extent the algebraic structure of a subgroup $G \subset \operatorname{Homeo}(M)$ influences or constrains the possible dynamics of actions of $G$ on $M$. In this way we can start to build a dictionary between algebraic properties such as nilpotency, torsion, subgroup distortion, amenability, and so on, and dynamical properties such as entropy, existence of fixed points, or stability under perturbation.

To give one further perspective, one can also (somewhat tautologically) view the groups $\operatorname{Homeo}(M)$ and $\operatorname{Diff}(M)$ as examples of automorphism groups of a locally homogeneous space-namely, the manifold $M$ itself, with its topological or smooth structure. But these groups also act transitively on many natural objects associated with $M$, especially in the case where $M$ is connected. These include the space of labeled or unlabeled $n$-tuples of points in the case where the manifold has dimension at least two, the space of ordered tuples in the 1-dimensional case, spaces of embedded discs, of simple closed curves (nonseparating simple closed curves when $M$ is a surface), and so on. As such, they are also groups of automorphisms of the configuration spaces of points, curves, discs, etc. The standard

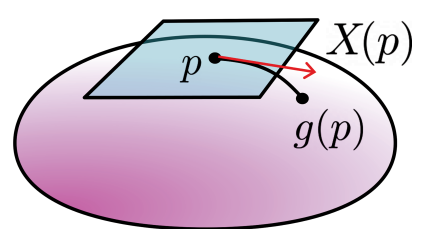

Figure 1. A local chart for $\operatorname{Diff}(M)$ to the space of vector fields on $M$.

compact-open or $C^{\infty}$ topology makes $\operatorname{Homeo}(M)$ and $\operatorname{Diff}(M)$ examples of Polish (completely metrizable) spaces, fostering some recent interactions with descriptive set theory, which has a rich toolkit to study Polish groups and automorphism groups of a wide class of structures.

\section{A Guiding Principle: Analogies with Simple Lie Groups}

The main barrier to understanding the groups $\operatorname{Homeo}(M)$ and $\operatorname{Diff}(M)$ is the fact that these groups are just intractably huge, even when $M$ itself is low-dimensional or otherwise of low complexity. For instance, fix some manifold $M$ of dimension at least 2. It is an open question whether there exists a finitely generated, torsion-free group that is not isomorphic to some subgroup of $\operatorname{Homeo}(M)$. This question has only recently been answered if you replace $\operatorname{Homeo}(M)$ with $\operatorname{Diff}(M)$; this is the solution to Zimmer's conjecture of Brown, Hurtado, and Fisher as explained in Fisher's recent article in this series [Fis20].

A helpful organizing principle is to think of homeomorphism and diffeomorphism groups as loose analogs of simple Lie groups. The group $\operatorname{Diff}(M)$ is in fact, in a precise sense, an infinite-dimensional Lie group with a Fréchet manifold structure, locally modeled on the infinite-dimensional vector space of smooth vector fields on $M$. A local chart from a neighborhood of the identity in $\operatorname{Diff}(M)$ to the space of vector fields is given by mapping a diffeomorphism $g$ to the vector field $X(p):=\exp _{p}^{-1} g(p)$, where $\exp _{p}$ is the Riemannian exponential map on a neighborhood of the identity in the tangent space $T_{p}(M)$. Charts based at other points than the identity element may be obtained by composing with left multiplication. This gives $\operatorname{Diff}(M)$ a smooth structure for which left multiplication and inverses are smooth maps, and its "Lie algebra" is the Lie algebra of vector fields on $M$.

However, the local chart above is not the usual Lie algebra exponential map! In fact, the Lie algebra exponentialassigning to a vector field the time-one map of the flow generated by this vector field-is not surjective onto any neighborhood of the identity, even in the simple case where $M=S^{1}$. (It is a pleasant exercise to identify some such diffeomorphisms that are not the time-one map of any flow. In the circle case these are diffeomorphisms with some, but not all, points being periodic with finite period greater than one. See also Milnor's wonderful survey [Mil84].) For 
this reason, the Lie group structure on $\operatorname{Diff}(M)$ is-perhaps counterintuitively-not usually the source of analogy with finite-dimensional simple Lie groups. Additionally, the group Homeo $(M)$ has no natural smooth structure or Lie algebra, but many of the known parallels between diffeomorphism groups and simple Lie groups also hold here. Rather, I believe that the important feature shared by all of these groups is the fact that they are the automorphism groups of highly homogeneous structures.

The remainder of this article is organized to illustrate specific instances of this analogy between simple (and occasionally semisimple) Lie groups and diffeomorphism groups, beginning with simplicity itself. To keep things within a reasonable scope, I have chosen to focus primarily on the identity components $\operatorname{Homeo}_{0}(M)$ and $\operatorname{Diff}_{0}(M)$ of the homeomorphism and diffeomorphism groups of a manifold $M$, as discussion of the mapping class groups Homeo( $M) / \mathrm{Homeo}_{0}(M)$ (and analogously for Diff) would take us much further afield.

\section{Simplicity}

Recall that a Lie group is simple if its Lie algebra is nonabelian and has no nontrivial proper ideals. The best ana$\log$ of this for topological groups is to ask whether the identity component of the group has no nontrivial proper normal subgroups. When $M$ is a noncompact manifold, the subgroup of compactly supported homeomorphisms or diffeomorphisms-those which pointwise fix the complement of some compact set-is an obvious normal subgroup, so the relevant object of study is its identity component, denoted $\operatorname{Homeo}_{c}(M)$ or $\operatorname{Diff}_{c}(M)$. Of course, when $M$ is compact, these are just the groups $\mathrm{Homeo}_{0}(M)$ and $\operatorname{Diff}_{0}(M)$ themselves.

In both the smooth and topological cases, simplicity (in the algebraic sense described above) was established in the 1970 s by Edwards, Kirby, Thurston, and others ${ }^{1}$ as a consequence of the following three general properties:

1. Fragmentation. Let $\mathcal{O}$ be an open cover of $M$. Then $\operatorname{Diff}_{c}(M)$ and $\mathrm{Homeo}_{c}(M)$ are generated by homeomorphisms supported on elements of $\mathcal{O}$.

2. Localized perfectness. Let $B$ be an open ball in $M$. Any element of $\operatorname{Diff}_{c}(B)$ or $\mathrm{Homeo}_{c}(B)$ can be written as a product of commutators.

3. Simplicity of the commutator group. Let $g \neq i d$ in $\operatorname{Diff}_{c}(M)$, and let $B \subset M$ be an open ball. Then any commutator in $\operatorname{Diff}_{c}(B)$ lies in the normal closure of $g$. The same holds replacing Diff with Homeo.

While I have deliberately stated these in parallel for Diff and Homeo, the proofs of 1 and 2 are entirely different in the smooth and $C^{0}$ case. Fragmentation is easy for

\footnotetext{
${ }^{1}$ This being an expository note, the number of citations in the text is limited. A version of this article with an extended reference list is available at https://e .math.corne11.edu/people/mann/homeo.pdf
}

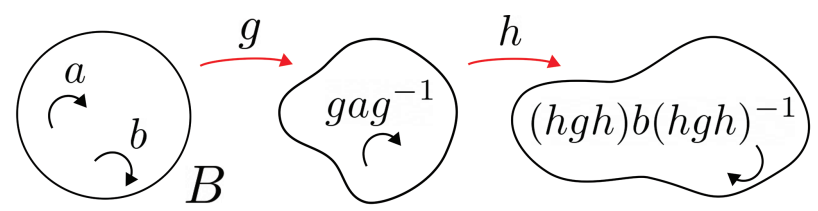

Figure 2. Schematic for the simplicity argument. The commutator $[a, b]$ can be written as $\left[[a, g],\left[b, h g h^{-1}\right]\right]$.

diffeomorphisms: an element of $\operatorname{Diff}_{c}(M)$ is always the time-one map of a compactly supported time dependent vector field, and cutting that vector field off by smooth bump functions is the only tool required for fragmentation. No such strategy works for homeomorphisms; rather, the proof is a deep result of Edwards and Kirby using the same machinery that goes into proving the annulus theorem.

Similarly, perfectness of $\operatorname{Homeo}_{c}(B)$ is an old trick (perhaps first appearing in this form in a paper of R. D. Anderson in 1958) but for $\operatorname{Diff}_{c}(B)$ it is a hard theorem of Thurston tied to the cohomology of classifying spaces, and it uses results of Herman for diffeomorphisms of the circle and the torus that rely heavily on KAM theory.

The common point is that localized perfectness implies simplicity. This is a short argument which uses a clever trick of "displacing supports." Given a homeomorphism $g$ (other than the identity), one wishes to show that any other homeomorphism $f$ can be written as a product of conjugates of $g$. I'll give the essence of the argument here, in the special case where $f$ has support on some small ball $B$ such that $g(B) \cap B=\emptyset$. If $g$ is not the identity, such a ball is guaranteed to exist, and stepping from here to the general case is not too difficult.

So suppose $f$ is such a homeomorphism, and that $f$ can be written as a commutator $f=a b a^{-1} b^{-1}$ (the argument is essentially the same if $f$ is a product of several commutators - this is where we are using local perfectness). Going forward, I'll use the standard notation $[a, b]$ for the commutator $a b a^{-1} b^{-1}$. Recall that $g(B) \cap B=\emptyset$. Take any homeomorphism $h$ that pointwise fixes $B$ and moves $g(B)$ disjoint from $B \cup g(B)$. One now checks by hand that $f=\left[[a, g],\left[b, h g h^{-1}\right]\right]$ which, if you write it out and unpack all the commutators, is easily seen to be a product of conjugates of $g$. The trick to showing that $f$ agrees with this nested commutator is the elementary fact that homeomorphisms with disjoint support commute. The conjugate $g a^{-1} g^{-1}$ has support on $g(B)$, so $[a, g]$ has support on $B \cup g(B)$ and agrees with $a$ on $B$. Similarly, $[b, h g h-1]$ agrees with $b$ on $B$ and has support on $B \cup h g(B)$. Thus, on $g(B)$ and on $h g(B)$ these two commutators commute, so $\left[[a, g],\left[b, h g h^{-1}\right]\right]$ is supported on $B$ and agrees with $[a, b]$ there. Variations, some quite sophisticated, of this trick of displacing supports to produce commuting elements appear throughout the literature cited below. 
Indicative of the subtleties involved in the question of simplicity, there is one case which we still do not know how to treat. In between $\operatorname{Homeo}_{c}(M)$ and $\operatorname{Diff}_{c}(M)$ lie the groups $\operatorname{Diff}_{c}^{r}(M)$ of $C^{r}$ diffeomorphisms for $r=1,2,3, \ldots$. Mather, also in the 1970s, showed with yet a different strategy that the groups $\operatorname{Diff}_{c}^{r}(M)$ are also perfect-except in the case $r=\operatorname{dim}(M)+1$, where it remains open! To this day, whether $\operatorname{Diff}_{0}^{2}\left(S^{1}\right)$ is a simple group remains an open question; the sticking point here is exactly the problem of local perfectness. Further references and discussion can be found in [Ban97].

\section{Automatic Continuity}

A broad question, applicable to any topological group, is to what extent does the algebraic structure of the group determine its possible group topologies?

Along this line, in the 1930s and 40s, Cartan, van der Waerden, Freudenthal, and others studied abstract homomorphisms between simple (and also semisimple) Lie groups. A sample consequence of their work is the following.

Theorem 0.1. An abstract algebraic isomorphism between compact semisimple Lie groups, or between absolutely simple real Lie groups, is necessarily continuous.

Further notable work in this direction was done by Borel and Tits in the 1970s. Much more recently, L. Kramer removed the hypothesis that the target group be a Lie group, showing the following.

Theorem 0.2 (Kramer [Kra11]). An abstract isomorphism between an absolutely simple real Lie group and a locally compact, $\sigma$-compact group is necessarily continuous.

As a consequence of this, one can show that the standard Lie group topology is the unique locally compact, $\sigma$ compact group topology on such a group (a result proved earlier for compact Lie groups by Kallman). The hypothesis "absolutely simple" prevents such counterexamples as a discontinuous algebraic automorphism of $\mathbb{R}$ obtained from a linear transformation of $\mathbb{R}$ as a vector space over $\mathbb{Q}$, or a discontinuous algebraic automorphism of $\mathrm{SL}_{n}(\mathbb{C})$ induced by a wild automorphism of $\mathbb{C}$. But these are, in a sense made precise by Kramer, the only kinds of pathologies that can occur.

Theorems 0.1 and 0.2 are instances of automatic continuity results, promoting an algebraic morphism to a topological one. While their proofs rely on Lie-theoretic techniques, Hurtado recently showed that a form of automatic continuity holds for diffeomorphism groups as well.

Theorem 0.3 (Hurtado [Hur15]). Let $M$ be a compact manifold, and $N$ any manifold. Any homomorphism $\Phi: \operatorname{Diff}_{0}(M) \rightarrow \operatorname{Diff}_{0}(N)$ is necessarily continuous.
Homeomorphism groups satisfy an even stronger version of automatic continuity, analogous to Kramer's result.

Theorem 0.4 (Rosendal, Solecki [RS07], Mann [Man16]). Let $M$ be a manifold, compact or homeomorphic to the interior of a compact manifold with boundary, and $G$ any separable topological group. Then any homomorphism $\Phi: \operatorname{Homeo}(M) \rightarrow G$ is necessarily continuous.

This says the algebraic structure of the group "remembers" the topology, in a very strong way. Separability of the target $G$ is needed as a hypothesis to eliminate obvious counterexamples such as the (discontinuous) identity map from Homeo $(M)$ (with its usual compact-open topology) to $\operatorname{Homeo}(M)$ equipped with the discrete topology.

Other examples of groups recently found to satisfy strong forms of automatic continuity include the group of an infinite-dimensional, separable, complex Hilbert space with the strong operator topology, the isometry group of the Urysohn universal metric space, and automorphism groups of Lebesgue probability spaces. (These are results of Tsankov, Sabok, and Ben Yaacov-Berenstein-Melleray, respectively.) Many of these results rely on the technique of ample generics in Polish groups developed by Kechris and Rosendal. Rosendal's survey [Ros09], though by now slightly out of date, is a good invitation to the subject.

While the statements of Theorems 0.3 and 0.4 mirror each other-taking $G=\operatorname{Homeo}(N)$ for some other manifold $N$ in the statement of Theorem 0.4 gives the $C^{0}$ counterpart of Hurtado's smooth version-as was the case for simplicity, the proofs of these two results are essentially different. The $C^{0}$ case, with the exception of the line and the circle, relies on a kind of diagonal argument common in these proofs for other automorphism groups, together with commutator tricks like the one in the proof of simplicity explained above. The smooth case uses the idea of averaging a metric under a sequence of diffeomorphisms to show that any sequence tending to the identity in $\operatorname{Diff}_{0}(M)$ converges along a subsequence to an isometry in $\operatorname{Diff}_{0}(N)$. This is then improved to convergence to the identity, from which one concludes continuity. Establishing such convergence requires maintaining control on derivatives of diffeomorphisms, motivating ideas that would later lead Hurtado to work on the Burnside problem for diffeomorphisms of the sphere, and the Zimmer program explained in the last section of this article.

Interestingly, automatic continuity (either in Hurtado's sense with a restricted target, or the very general sense) for the groups $\operatorname{Diff}_{0}^{r}(M)$ when $0<r<\infty$ remains open. As some positive evidence towards this, a result of Kallman from the 1980s states that the $C^{r}$ topology is the unique Polish topology on $\operatorname{Diff}^{r}(M)$. This implies in particular that there can be no discontinuous automorphisms of such a group. But in fact, a much stronger statement about 
automorphisms was known even earlier: any automorphism of $\operatorname{Diff}^{r}(M)$ is inner. This kind of rigidity of the group structure is best framed as a "reconstruction" result, which brings us to our next topic.

\section{Reconstruction Theorems}

While automatic continuity says that the algebraic structure of a homeomorphism group "remembers" its group topology, it was known decades earlier that the algebraic structure remembers the underlying manifold. This is a 1963 result of Whittaker, which states that the existence of an abstract isomorphism $\Phi: \mathrm{Homeo}_{0}(M) \rightarrow \mathrm{Homeo}_{0}(N)$ implies that $M$ is homeomorphic to $N$ and $\Phi$ is induced by conjugation by a homeomorphism.

Whittaker's work also applies to more general topological spaces, and does not require that manifolds be compact. However, some care is needed in the hypotheses because (for example) $\mathrm{Homeo}_{0}(\mathbb{R})$ is abstractly isomorphic to the homeomorphism group of a closed interval-one simply forgets the endpoints of the closed interval and restricts the homeomorphisms to the interior-but of course the line and the interval are not homeomorphic.

Nearly twenty years later, Filipkiewicz extended this to diffeomorphism groups, showing that in this case the algebraic structure of the group also remembers the regularity of the diffeomorphisms. This gives us the "infinite family of desserts" advertised above.

Theorem 0.5 (Filipkiewicz [Fil82]). Let $M$ and $N$ be compact manifolds and suppose there is an isomorphism $\Phi: \operatorname{Diff}_{0}^{r}(M) \rightarrow \operatorname{Diff}_{0}^{s}(N)$. Then $r=s$, the manifolds $M$ and $N$ are $C^{r}$ diffeomorphic, and $\Phi$ is induced by a $C^{r}$ diffeomorphism.

In both this and Whittaker's theorem, the basic strategy is to promote an isomorphism between transformation groups to a bijection between manifolds by associating subgroups of $\operatorname{Diff}^{r}(M)$ or $\mathrm{Homeo}_{0}(M)$ to subsets of $M$, eventually showing that the isotropy (point stabilizer) subgroup $G_{x}$ of a point $x \in M$ is mapped under an isomorphism $\Phi$ to the isotropy subgroup of some point of $N$. A key tool is commutation relations. To give a toy example, note that $G_{x}$ is characterized by the following property. A nontrivial diffeomorphism $g$ lies in $G_{x}$ if and only if, for any open set $B$ containing $x$, the subgroup of diffeomorphisms supported on $B$ does not commute with its conjugate by $g$. Though this is far from a proof that point stabilizers are mapped to point stabilizers, it is a hint that one might be able to pick out such subgroups by algebraic relations (in this case, commutation).

The relationship between point stabilizers in $M$ and those in $N$ gives a point-to-point map $\tau: M \rightarrow N$. Furthermore, this map is compatible with the group action in the sense that, for any $f \in \operatorname{Diff}_{0}^{r}(M)$, we have $G_{f(x)}=f\left(G_{x}\right) f^{-1}$ from which it follows that $\tau(f(x))=\Phi(f) \tau(x) \Phi(f)^{-1}$. This compatibility allows one to show fairly easily that $\tau$ is a homeomorphism; Filipkiewicz then appeals to deep results of Montgomery and Zippin [MZ55] to conclude that it is a $C^{r}$ diffeomorphism.

Analogous theorems for groups of symplectomorphisms, contact diffeomorphisms, and volume-preserving diffeomorphisms were later obtained by Banyaga. An expository account of this and Filipkiewicz's theorem can be found in [Ban97]. M. Rubin [Rub89] generalized Whittaker's results in a different direction, extending it to more general classes of topological spaces and subgroups of their automorphism groups using a more model-theoretic framework. Both show that the algebraic structure of the automorphism group is sufficient to recover the underlying manifold or space.

Such reconstruction results can be interpreted as instances of the broad principle that any sufficiently rich object can be recovered, within its class, from its automorphism group. To give a sense of the breadth of this principle, let me mention two other recent examples (with a geometric topological flavor; apologies for my bias). The first is the folklore theorem that the homeomorphism class of a compact surface $S$ can, with a few low-complexity exceptions, be completely recovered from the mapping class group $\operatorname{Homeo}(S) / \mathrm{Homeo}_{0}(S)$ of homeomorphisms up to isotopy. This was recently extended by Bavard-DowdallRafi to infinite-type noncompact surfaces as well. The second example that comes to mind is a recent result of Cantat that Cremona groups of projective spaces determine the dimension of the space. Surely the reader can draw a few examples from their own field of study!

\section{Optimal Regularity}

Filipkiewicz's theorem says that $\operatorname{Diff}_{0}^{r}(M)$ and $\operatorname{Diff}_{0}^{s}(M)$ are nonisomorphic when $r \neq s$. But it leaves no practical way to distinguish them algebraically. As a concrete step in this direction, one could ask:

For a given manifold $M$, can the groups $\operatorname{Diff}_{0}^{r}(M)$ and $\operatorname{Diff}_{0}^{S}(M)$ be distinguished by their finitely generated subgroups?

Navas posed this question explicitly for onedimensional manifolds in a 2017 problems list [Nav18]. As he notes, questions of distinguishing regularity have a long history among dynamicists that can be traced back to work of Denjoy on the relationship between regularity and topological dynamics. Denjoy showed that, while there are many $C^{1}$ counterexamples, if $f$ is any $C^{2}$ diffeomorphism of the circle without periodic or fixed points, then for every point $x$, the orbit $\left\{f^{n}(x): n \in \mathbb{Z}\right\}$ is a dense subset of the circle. A typical $C^{1}$ counterexample is illustrated in Figure 3: there is an invariant Cantor set (red in the figure) on which the diffeomorphism behaves like a rotation. To visualize the dynamics, the figure shows the mapping torus 


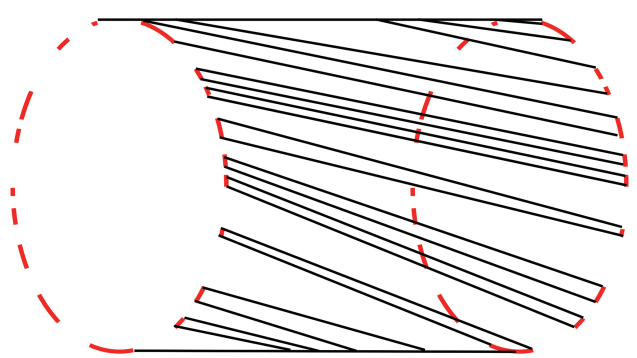

Figure 3. Dynamics of a $C^{1}$ "Denjoy counterexample" diffeomorphism of the circle.

of such a homeomorphism, with points on the leftmost circle connected to their images on a copy of the circle to the right. Dense orbits and existence or nonexistence of periodic points are both properties invariant under conjugation by homeomorphisms, so Denjoy's theorem implies that there exist $C^{1}$ diffeomorphisms which cannot be topologically conjugate to any $C^{2}$ diffeomorphism, an observation later generalized by Harrison to higher dimensional manifolds and higher regularity.

Denjoy's theorem takes as input a weak dynamical assumption (no periodic points) and a regularity hypothesis (twice continuously differentiable), and gives as output the strong dynamical conclusion that all orbits are dense. Thus, it reveals something about the interplay between topological dynamics and regularity. When one has a group action rather than a single diffeomorphism, the algebraic structure of the group can also enter the picture. A well-known and early instance of a result along these lines is Thurston's generalization of the Reeb stability theorem. Thurston's theorem says that a group of $C^{1}$ diffeomorphisms of a manifold with a common fixed point at which the diffeomorphisms are all first order equal to the identity map (that's a regularity + dynamical assumption) necessarily has the (algebraic) local indicability property that every finitely generated subgroup surjects to $\mathbb{Z}$. The search for similar theorems of the form

$$
\text { [dynamical assumptions] }+ \text { [regularity] }
$$$$
\Rightarrow \text { [algebraic conclusion] }
$$

(or any permutation thereof) remains an active and exciting area. This is the context for Navas' question above.

Several results along these lines are known to hold when $M$ is one-dimensional, and a positive answer to Navas' question was given by Kim and Koberda not long after the appearance of his problems list. Their proof uses a highly refined version of the diffeomorphisms with disjoint supports commute line of tricks to encode the regularity of a diffeomorphism by the rate at which it moves, and therefore contracts, small subintervals (forced to be supports of group elements) towards a fixed point. Obtaining enough control on supports to pull off this argument from purely algebraic hypotheses is quite a technical feat, and even so, in low regularity they use some additional dynamical input from earlier work of Bonatti, Monteverde, Navas, and Rivas. A different approach to this question, inspired by work of Ghys, was pursued by the author and Wolff, with the aim of eventually addressing the question for higher dimensional manifolds. While we found a short proof somewhat complimentary in technique to that of Kim-Koberda, the higher dimensional case is still out of reach!

\section{Structure Theorems for Group Actions}

The reconstruction results of the previous sections came out of a classification of isomorphisms between large transformation groups. Classifying homomorphisms between them is a significantly harder problem. A homomorphism $\mathrm{Homeo}_{0}(M) \rightarrow \operatorname{Homeo}(N)$ is simply a group action of the group $\mathrm{Homeo}_{0}(M)$ on the space $N$, so going forward I will use the language of group actions to describe such maps. (Again, we are restricting to the identity component $\mathrm{Homeo}_{0}(M)$ to avoid actions that factor through an action of the mapping class group.)

There are many natural examples of such actions. The groups $\operatorname{Homeo}_{0}(M)$ and $\operatorname{Diff}_{0}(M)$ obviously act on the product manifold $M \times W$ for any manifold $W$. They also have a natural action on

$$
\operatorname{Conf}_{k}(M)=((M \times \cdots \times M)-\triangle) / \operatorname{Sym}_{n},
$$

the "configuration space" of $k$ distinct, unlabeled points in $M$. In some instances, such as when $M$ is a Riemannian manifold of negative curvature, or more generally when $\pi_{1}(M)$ has trivial center, the action of $\operatorname{Homeo}_{0}(M)$ or $\operatorname{Diff}_{0}(M)$ on $M$ can also be shown to lift to an action on covers of $M$. Finally, to give some examples special to the differentiable case, $\operatorname{Diff}_{0}(M)$ also acts naturally on the tangent bundle of $M$, the projectivized tangent bundle of $M$, and various jet bundles over $M$. With the exception of the product action on $M \times W$ and the tangent bundle action, all of the examples above have a single orbit, indicating that even a classification of transitive actions is a nontrivial and potentially difficult problem.

This classification problem has been posed, in various forms, at least since the time of Whittaker's work. Part of the challenge in the problem is simply stating a clear conjectural picture. Rubin, in the 1989 article [Rub89] briefly mentioned above, asks "Are there any reasonable assumptions on the type of the embeddings so that the embeddability of $\operatorname{Homeo}(X)$ in $\operatorname{Homeo}(Y)$ will imply that $X$ is some kind of continuous image of $Y$ ?" (emphasis mine). Embeddability here is meant in the algebraic sense of the existence of an injective homomorphism $\operatorname{Homeo}(X) \rightarrow \operatorname{Homeo}(Y)$, and Rubin is speaking not just of manifolds, but of quite general topological spaces. Two years after that, Ghys 
asked whether embeddability of $\operatorname{Diff}_{0}(M)$ into $\operatorname{Diff}_{0}(N)$ implies that the dimension of $N$ must be at least as large as that of $M$. This was answered positively by Hurtado in 2015 using his automatic continuity result [Hur15], improving on a very low-dimensional case I had proved earlier (but that case did not have automatic continuity!). Hurtado also gives a more precise picture of what a classification might look like. Although he does not give an explicit conjecture, he notes that $\operatorname{Diff}_{0}(M)$ acts naturally on the tangent bundle of $M$ (as well as various Grassmanians, etc.) and remarks that all known examples of actions of $\operatorname{Diff}_{0}(M)$ on other manifolds are built from pieces that have the form of natural bundles over $M$ or configuration spaces of points on $M$. If one believes this is an exhaustive list, then one could refine Rubin's question to ask for a stratification of the target manifold into understandable pieces that map continuously either to $M$ or to tuples of points on $M$.

Pursuing the analogy between large transformation groups and Lie groups, one could frame the problem of classifying morphisms between such groups as the analog of representation theory for these groups-a perspective which I myself have advertised. But a more useful framing is to draw parallels with the classical theory of compact (or locally compact) transformation groups pursued by Bredon, Conner, Floyd, Montgomery, Zippin, and others in the 1950s-70s. Montgomery and Zippin [MZ55] frame one aspect of this program as a search for the similarities between actions of a compact group on a manifold and standard linear or other model geometric actions. Years later, Bredon [Bre72], in broad terms, says that he views theory of compact transformation groups as "a generalization of the theory of fiber bundles." ${ }^{2}$ Indeed, the general starting point for this theory is typically to first show that only finitely many orbit types may occur, then to understand and classify orbits, and then to say that the neighborhood of any orbit looks something like a direct product, giving a local bundle-like structure. However, complete solutions to the classification problem for compact group actions are generally only attainable with some restriction on the types of orbits that appear, whether by explicit assumption, or by restricting the dimension of the space and the group. A comprehensive introduction to such problems can be found in Bredon's book [Bre72].

That one might similarly be able to classify actions of homeomorphism or diffeomorphism groups is a comparatively recent idea, perhaps first advocated for by Ghys, although I personally learned this question from Benson Farb. However, it was automatic continuity theorems that

\footnotetext{
${ }^{2}$ While [MZ55, Bre72] are old references, the study of transformation groups has fractured into many different directions since then. Terry Tao's Hilbert's Fifth Problem and Related Topics covers a good deal of the classical material from a modern perspective, and is another good starting point.
}

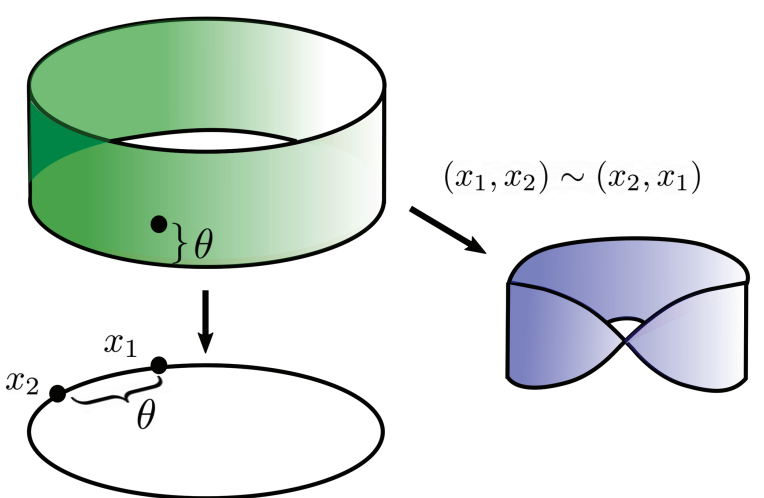

Figure 4. The configuration space of two labeled points on $S^{1}$ is an open annulus, that of two unlabeld points is topologically a mobius band. Both inherit an action of Homeo $_{0}\left(S^{1}\right)$.

provided the catalyst needed to make major progress in this direction. Using automatic continuity for actions of $\operatorname{Homeo}\left(S^{1}\right)$, in $2012 \mathrm{E}$. Militon gave a complete classification of actions of $\mathrm{Homeo}_{0}\left(S^{1}\right)$ on the closed annulus and 2torus. His classification gives a decomposition of the torus or annulus into orbits, each homeomorphic to either the circle with the standard action, or to an open annulus with the action coming from identifying the annulus with the configuration space of two (labeled) points in $S^{1}$. See Figure 4 . These two types of orbits may be glued together in a multitude of different ways, leading to an uncountable family of nonconjugate examples which Militon describes completely.

Recent work of Lei Chen and the author gives a general classification of orbit types in all dimensions.

Theorem 0.6 (Chen-Mann [CM]). Suppose that $\mathrm{Homeo}_{0}(M)$ acts on a manifold $N$. Then each orbit is the continuous, injective image of a cover of a configuration space $\operatorname{Conf}_{n}(M)$.

While locally embedded, such orbits are not necessarily globally embedded. The simplest example where this fails is the following bundle construction.

Example 0.7. Suppose $\Sigma$ is a surface of genus at least two. Then $\mathrm{Homeo}_{0}(\Sigma)$ lifts to act continuously, by homeomorphisms on the universal cover $\widetilde{\Sigma}$, which is topologically an open disc, and this action commutes with the action of the deck group. Extend this to an action on $\widetilde{\Sigma} \times S^{1}$ by homeomorphisms that are constant on the second factor. Orbits of this action are embedded open discs, but we can change this by passing to a quotient of the product space by an action of $\pi_{1}(\Sigma)$ that is nontrivial on the second factor. To be precise, the fundamental group $\pi_{1}(\Sigma)$ admits many actions by homeomorphisms on the circle-for instance, any hyperbolic structure on the surface gives a discrete, faithful representation of $\pi_{1}(\Sigma)$ into $\operatorname{PSL}(2, \mathbb{R})$ which acts naturally on $\mathbb{R} P^{1} \cong S^{1}$ by fractional linear transformations. 


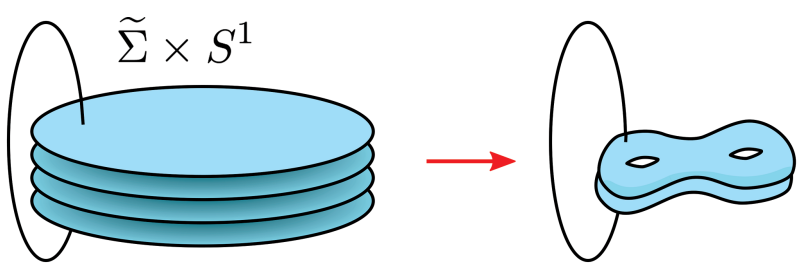

Figure 5. A quotient of $\widetilde{\Sigma} \times S^{1}$ by a diagonal action of $\pi_{1}(\Sigma)$ is a circle bundle over $\Sigma$ which inherits an action of $\mathrm{Homeo}_{0}(\Sigma)$. The trivial action on $S^{1}$ gives a product bundle with closed orbits as shown, while typical actions have dense orbits.

Fix such an action, and take the quotient of $\widetilde{\Sigma} \times S^{1}$ by the diagonal action of $\pi_{1}(\Sigma)$ by deck transformations on the first factor, and homeomorphisms of $S^{1}$ on the second. The result is an $S^{1}$ bundle over $\Sigma$ with a continuous action of $\mathrm{Homeo}_{0}(\Sigma)$ by homeomorphisms. Provided the action of $\pi_{1}(\Sigma)$ on $S^{1}$ doesn't factor through that of a finite group, the orbits of the $\mathrm{Homeo}_{0}(\Sigma)$ action on this quotient space will accumulate on themselves.

Interestingly, if one takes the representation of $\pi_{1}(\Sigma)$ into $\operatorname{PSL}(2, \mathbb{R})$ described above, then the resulting circle bundle over $\Sigma$ is, topologically, the unit tangent bundle (or projectivized tangent bundle) of the surface. While the group of diffeomorphisms of the surface has an obvious action on this space, I find it quite surprising that the group of homeomorphisms also does since a homeomorphism has no obvious induced action on the tangent space to a point!

Because of automatic continuity, Theorem 0.6 reduces to the problem of classifying certain closed subgroups of $\mathrm{Homeo}_{0}(M)$. (In the case where $M$ is noncompact, automatic continuity does not quite apply and a little more work is needed, so for simplicity I'll assume here that $M$ is compact.) If $\rho: \operatorname{Homeo}_{0}(M) \rightarrow \operatorname{Homeo}(N)$ is any action, then the stabilizer

$$
G_{y}:=\left\{g \in \operatorname{Homeo}_{0}(M): \rho(g)(y)=y\right\}
$$

of some point $y \in N$ is a closed subgroup, and the orbit map gives a continuous, injective map of the coset space $\operatorname{Homeo}_{0}(M) / G_{y}$ into $N$. Thus, Theorem 0.6 becomes equivalent to determining which closed subgroups of $\mathrm{Homeo}_{0}(M)$ are "large enough" so that their coset space can be locally embedded in a finite-dimensional manifold. Classical invariance of domain says that $\mathbb{R}^{n}$ is not locally embeddable in a manifold of dimension less than $n$, so no $\mathbb{R}^{n}$ subgroup of $\operatorname{Homeo}_{0}(M)$, that is to say no $n$-many commuting flows, can survive the quotient map to $\mathrm{Homeo}_{0}(M) / G_{y}$ if $\operatorname{dim}(N)<n$. Applying this observation to subgroups of $\mathrm{Homeo}_{0}(M)$ with disjoint supports, and quoting local simplicity, with enough work we eventually conclude that $G_{y}$ contains the identity component of the stabilizer of a finite set of points in $M$, hence Homeo $_{0}(M) / G_{y}$ is (up to taking a cover) identified with the configuration space of those points.
The example of $\operatorname{Diff}_{0}(M)$ acting on the projectivized tangent bundle of $M$ shows that a similar classification for actions of diffeomorphism groups, even for actions on compact manifolds, must account for more kinds of orbit types. Remarkably, this kind of construction-taking quotients of jets of diffeomorphisms up to $r$ th-order-is the only kind of new phenomenon that occurs: we show in $[C M]$ that if $\operatorname{Diff}_{0}(M)$ acts on a manifold by smooth diffeomorphisms, then every orbit is the continuous, injective image of a fiberwise quotient of the $r$-jet bundle over a cover of a configuration space of points on $M$.

Theorem 0.6 confirms the picture suggested by Hurtado [Hur15] that $N$ should be "built from pieces" that look like bundles-the pieces are simply the orbits of the action. Classifying all actions now amounts to understanding how such pieces can be glued together. We obtain some preliminary results under restrictions on dimension that simplify the types of orbits that may occur. (Such hypotheses are commonplace in the classical literature on compact transformation groups.) A sample theorem, paralleling classical "slice" or local product theorems for the compact group case, is as follows.

Theorem 0.8 (Chen-Mann [CM]). If $M$ and $N$ are connected manifolds with $\operatorname{dim}(N)<2 \operatorname{dim}(M)$ and $\mathrm{Homeo}_{c}(M)$ acts on $N$ without global fixed points, then $N$ has the structure of a generalized flat bundle over $M$, i.e., there is a homology manifold $F$ such that $N \cong(\widetilde{M} \times F) / \pi_{1}(M)$, where $\pi_{1}(M)$ acts diagonally by deck transformations on $\widetilde{M}$ and on $F$ by some representation to $\operatorname{Homeo}(F)$.

In fact, we can also describe the possible actions on $N$ up to conjugacy: they are all obtained by a construction as in Example 0.7. A related result for diffeomorphism groups of compact manifolds can be obtained without the hypothesis that the action has no global fixed points; one shows directly that in this setting no orbits can be singletons. This line of work also allows one to complete the classification of $\mathrm{Homeo}_{0}\left(S^{1}\right)$ actions on surfaces that was initiated by Militon, filling in the case of the 2-sphere and open annulus. However, it appears we have only yet seen the tip of the iceberg, and I hope to see much exciting work in this direction in the near future.

\section{Local and Global Rigidity of Lattices}

Having advocated for the analogy between Lie groups and diffeomorphism or homeomorphism groups, this survey would be incomplete without a discussion of the role of lattices in these groups.

A lattice $\Gamma$ in a Lie group $G$ is a discrete subgroup such that $G / \Gamma$ has finite volume with respect to the natural leftinvariant volume form on $G$ giving Haar measure. A lattice is called cocompact or uniform if $G / \Gamma$ is additionally compact. In one sense, lattices are small subgroups, being discrete and, under appropriate hypotheses on $G$, they 
are also always finitely generated groups. At the same time, that $G / \Gamma$ is finite volume means that lattices are also large and this gives them some remarkable rigidity properties, constraining the ways they may be embedded into $G$ and into other Lie groups. The paradigm example of such a result is Margulis' superrigidity theorem, which essentially says that the representation theory of a lattice $\Gamma$ in a semisimple Lie group $G$ is the same as the representation theory of $G$ itself: with a few technical caveats, any representation of $\Gamma$ to a finite-dimensional linear group extends to a continuous representation of $G$.

Infinite-dimensional linear representations of lattices have no such rigidity property. Remarkably, nonlinear representations-that is, representations of lattices to groups of diffeomorphisms-do. That this might be true was an idea first suggested by R. Zimmer in the late 1970s. Zimmer generalized other work of Margulis (specifically, on cocycle rigidity for group actions) to actions of lattices by measure-preserving diffeomorphisms of manifolds. He followed this with a series of broad conjectures about how lattices should act—or fail to act! —on manifolds, giving rise to what is now known as the Zimmer program. This program is often summarized by the catchphrase "big groups don't act on small manifolds" meaning that lattices in semisimple Lie groups that are large (specifically, of high rank) should not act faithfully on manifolds of low dimension.

More broadly, the Zimmer program aims to classify all actions of higher rank semisimple Lie groups and their lattices on compact manifolds, the idea being that they should be constrained to a collection of natural or obvious examples (such as, but not exclusively, those coming from actions of the ambient Lie group as is the case in Margulis' theorem). While a complete classification is a lofty and probably unattainable goal, there have been a number of remarkable recent advances, for which I refer the reader to [Fis20] and Fisher's other survey articles referenced there. Here I will only very briefly highlight one feature of this program, namely rigidity of geometric examples. This is distinct from the "big groups don't act on small manifolds" tagline, looking instead for examples of groups that $d o$ act on spaces, but whose possible actions are highly constrained. Of course, one expects techniques that answer this problem to apply to the "groups that don't act" program as well, and vice versa.

As one such example, consider the special linear group $\operatorname{SL}(n, \mathbb{Z})$, a nonuniform lattice in $\operatorname{SL}(n, \mathbb{R})$. Since the action of $\operatorname{SL}(n, \mathbb{Z})$ on $\mathbb{R}^{n}$ preserves the points with integer coordinates, it descends to an action on the torus $\mathbb{R}^{n} / \mathbb{Z}^{n}$, which preserves both Lebesgue measure and the natural affine structure on the torus-instances of what I mean by a "geometric example." These linear actions also have a strong dynamical property called Anosov dynamics: for

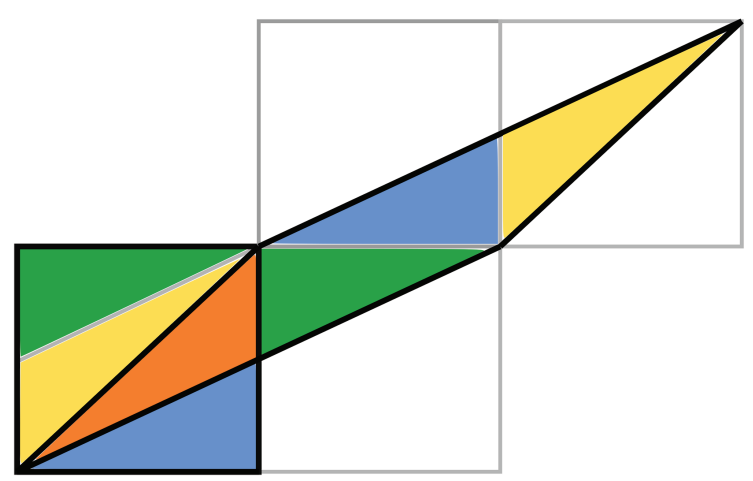

Figure 6. The action of $\left(\begin{array}{ll}2 & 1 \\ 1 & 1\end{array}\right) \in \mathrm{SL}(2, \mathbb{Z})$ on $\mathbb{R}^{2} / \mathbb{Z}^{2}$. The figure shows a fundamental domain and its image, translated back onto the fundamental domain.

any element with all eigenvalues off the unit circle, the tangent space of the manifold splits globally into invariant directions (here, eigendirections) that are contracted or expanded by iterates of the map. ${ }^{3}$

The most famous Anosov diffeomorphism is probably $\left(\begin{array}{ll}2 & 1 \\ 1 & 1\end{array}\right) \in \mathrm{SL}(2, \mathbb{Z})$, also known as Arnold's "cat map" after an illustration in Arnold's Ergodic Problems in Classical Mechanics in which he applies the map to a cartoon image of a cat. After several iterations, the cat's face is unrecognizably stretched and distributed around the torus. This is a basic property of Anosov maps. Formally speaking, volumepreserving Anosov diffeomorphisms are ergodic. One consequence of this is what one might call chaotic behavior: nearby points in the domain have vastly different trajectories under iterates of the map.

Despite this chaos on the level of trajectories of individual points, Anosov diffeomorphisms exhibit remarkable global stability, in the sense that if you nudge the whole system, the result is a system that is qualitatively exactly the same-typically (depending on the regularity involved) conjugate to the original system. These rigiditytype results for diffeomorphisms and 1-parameter families of diffeomorphisms date back to work of Anosov himself in the 1960s.

In the context of group actions, for groups other than $\mathbb{R}$ or $\mathbb{Z}$, in many cases Anosov dynamics of a single group element can be promoted via an understanding of the group structure to not only local rigidity but global rigidity for the whole action. One important and influential instance of this is in the work of Katok, Lewis, Zimmer, and others, starting in the 1990s. To give a sample result, Katok, Lewis, and Zimmer showed that any measure-preserving action of $\operatorname{SL}(n, \mathbb{Z})$ on $\mathbb{R}^{n} / \mathbb{Z}^{n}$ such that a single diffeomorphism has Anosov dynamics is (up to passing to a finite index subgroup) smoothly conjugate to a linear action. Some mild hypothesis is required on the measure, although in

\footnotetext{
${ }^{3}$ I am sweeping a few things under the rug here; the precise definition of Anosov is a formalization of this idea of invariant splitting.
} 
other work this hypothesis has been replaced with other hypotheses such as the existence of a finite orbit or a fixed point. See Spatzier's survey [Spa04] for a nice introduction to the history of such results.

Rigidity of lattices remains an active and exciting area. Recently, Brown, Rodriguez-Hertz, and Wang proved a much broader theorem along the lines of that of KatokLewis-Zimmer described above, but applicable to a much wider class of lattices and with weaker hypotheses. As well as obtaining global rigidity for actions of $\operatorname{SL}(n, \mathbb{Z})$ on tori $\mathbb{R}^{m} / \mathbb{Z}^{m}$ (note that $m$ is not required to equal $n$ here!) under suitable hypotheses, they obtain rigidity of cocompact lattices on nilmanifolds, a class which includes tori.

Theorem 0.9 (Brown-Rodriguez-Hertz-Wang [BRHW17]). Suppose $\Gamma$ is an irreducible cocompact lattice in a semisimple Lie group acting on a nilmanifold $M$ by smooth diffeomorphisms. If some element has Anosov dynamics, then (up to possibly passing to a finite index subgroup) this action is smoothly conjugate to a group of linear automorphisms of $M$.

Without passing to a finite index subgroup, one obtains conjugacy to an affine action-still a geometric example. Their work also covers nonirreducible and many nonuniform lattices, but in the interest of simplicity of the statement I have restricted to this case. However, differentiability is an essential component of this result. While Brown, Rodriguez-Hertz, and Wang can reduce the hypotheses from smooth to $C^{1}$ or even Lipschitz actions at the cost of a weaker conclusion, the machinery going into the regularity of the conjugacy is essentially a smooth phenomenon.

That said, there are also a few known instances of "rigidity of geometric examples" for groups acting by homeomorphisms. Although the definition of Anosov is in terms of the induced action of a diffeomorphism on the tangent bundle, and hence requires an action to be $C^{1}$, there are topological analogs of Anosov flows; the idea being that you can quantify the rate of expansion and contraction of continuous trajectories even if you cannot meaningfully speak of a splitting of the tangent space. These topological flows have many of the same dynamical properties, and $C^{0}$ perturbations of Anosov diffeomorphisms also exhibit similar stability properties.

However, Zimmer's conjecture that there should be "no actions of large groups on small manifolds," speaking here of discrete groups, is wide open in the $C^{0}$ case, except when the manifold is very small: dimension one. These onedimensional results rely on the total ordering of points on the line, or cyclic ordering of points on the circle, a property that can actually be promoted to a left-invariant total ordering on any group of homeomorphisms of the line or circle. As you might expect, such a total order on a group imposes strong algebraic constraints, and this has been successfully leveraged to show certain groups, including lattices, do not act. Morris' paper [Mor11] is a nice introduction; the state of the art can be found in a very recent paper of Deroin and Hurtado, who show nonorderability of all higher rank lattices.

There are also a few instances of $C^{0}$ rigidity of geometric examples in low dimension, even without Anosov dynamics. It is less clear what "geometric" should mean for a group acting by homeomorphisms, but one possible definition, in the style of a model geometry in the sense of Felix Klein, is that the action should be conjugate to that of a cocompact lattice in a Lie group which acts transitively on the manifold. With this definition, classifying geometric subgroups of $\mathrm{Homeo}_{0}\left(S^{1}\right)$ is an easy exercise: any group acting geometrically is (up to taking a finite, cyclic extension) the fundamental group $\pi_{1}\left(\Sigma_{g}\right)$ of a higher genus surface, acting on the circle via a discrete, faithful representation into $\operatorname{PSL}(2, \mathbb{R})$ or a finite, cyclic extension of $\operatorname{PSL}(2, \mathbb{R})$. Wolff and I showed these are precisely the rigid examples.

Theorem 0.10 (Mann, Mann-Wolff [MW]). All geometric actions of $\pi_{1}\left(\Sigma_{g}\right)$ are rigid, in the sense that every continuous deformation of such an action is semiconjugate to the original action. Conversely, any action of $\pi_{1}\left(\Sigma_{g}\right)$ on the circle that is rigid under all continuous deformations is necessarily semiconjugate to a geometric example.

Semiconjugate here means that there is a continuous, surjective, degree one map $h: S^{1} \rightarrow S^{1}$ intertwining the geometric action with its deformation. Deformation is meant in the large or global sense, in fact the whole connected component of a geometric action in the space of all homomorphisms of $\pi_{1}\left(\Sigma_{g}\right)$ into $\mathrm{Homeo}_{0}\left(S^{1}\right)$ consists precisely of the representations semiconjugate to it. The reason I mention this here is the suspicion that rigidity of geometric examples-perhaps with a different definition of geometric; I am not convinced we have the right one-should continue to be a fruitful future direction.

One phenomenon at play in the rigidity of geometric actions on the circle is a weak topological replacement of the rigidity of Anosov diffeomorphisms mentioned above. In the Anosov case, a single diffeomorphism has a global expansion/contraction property coming from a splitting of the tangent space at every point. Geometric actions of surface groups on the circle exhibit a contrasting notion of "expansion/contraction everywhere" that can be phrased in purely topological terms: each homeomorphism $f$ in the action has a finite set of attractors (points $x$ with a neighborhood $U$ such that the intersection of iterates $f^{n}(U)$, $n \geq 0$, is equal to $x$ ), and the union of attractors, taken over all group elements, forms a dense subset of the circle. That local dynamics of many group elements can stand in place of global dynamics of a single group element is far from a new idea, but is still a fruitful one. J. Bowden and I recently extended some of the ideas to prove a weaker, 
local $C^{0}$ rigidity for other geometrically motivated group actions on higher dimensional spheres, but how far this idea can be pushed is completely up in the air. I suspect, as many others do, that our guiding analogy with Lie groups holds true and Zimmer's philosophy applies quite broadly to actions of lattices by homeomorphisms on manifolds, but we are very far from understanding how this might play out.

\section{References}

[Ban97] Augustin Banyaga, The structure of classical diffeomorphism groups, Mathematics and its Applications, vol. 400, Kluwer Academic Publishers Group, Dordrecht, 1997. MR1445290

[Bre72] Glen E. Bredon, Introduction to compact transformation groups, Pure and Applied Mathematics, vol. 46, Academic Press, New York-London, 1972. MR0413144

[BRHW17] Aaron Brown, Federico Rodriguez Hertz, and Zhiren Wang, Global smooth and topological rigidity of hyperbolic lattice actions, Ann. of Math. (2) 186 (2017), no. 3, 913-972, DOI 10.4007/annals.2017.186.3.3. MR3702679

[CM] Lei Chen and Kathryn Mann, Structure theorems for actions of homeomorphism groups, arXiv:1902.05117[math.GT], 2019.

[Fil82] R. P. Filipkiewicz, Isomorphisms between diffeomorphism groups, Ergodic Theory Dynam. Systems 2 (1982), no. 2, 159-171 (1983), DOI 10.1017/s0143385700001486. MR693972

[Fis20] David Fisher, Recent developments in the Zimmer program, Notices Amer. Math. Soc. 67 (2020), no. 4, 492-499.

[Hat12] Allen Hatcher, A 50-year view of diffeomorphism groups, 2012, Slides from a talk at the 50th Cornell Topology Festival. Available at pi . math. corne11. edu/ hatcher /Papers/Diff(M)2012.pdf.

[Hur15] Sebastian Hurtado, Continuity of discrete homomorphisms of diffeomorphism groups, Geom. Topol. 19 (2015), no. 4, 2117-2154, DOI 10.2140/gt.2015.19.2117 MR3375524

[Kra11] Linus Kramer, The topology of a semisimple Lie group is essentially unique, Adv. Math. 228 (2011), no. 5, 2623-2633, DOI 10.1016/j.aim.2011.07.019. MR2838051

[Man16] Kathryn Mann, Automatic continuity for homeomorphism groups and applications, Geom. Topol. 20 (2016), no. 5, 3033-3056, DOI 10.2140/gt.2016.20.3033. With an appendix by Frédéric Le Roux and Mann. MR3556355

[MW] Kathryn Mann and Maxime Wolff, Rigidity and geometricity for surface group actions on the circle, arXiv:1710.04902 [math.GT].

[Mil84] J. Milnor, Remarks on infinite-dimensional Lie groups, Relativity, groups and topology, II (Les Houches, 1983), North-Holland, Amsterdam, 1984, pp. 1007-1057. MR830252

[MZ55] Deane Montgomery and Leo Zippin, Topological transformation groups, Interscience Publishers, New YorkLondon, 1955. MR0073104
[Mor11] Dave Witte Morris, Can lattices in $\operatorname{SL}(n, \mathbb{R})$ act on the circle?, Geometry, rigidity, and group actions, Chicago Lectures in Math., Univ. Chicago Press, Chicago, IL, 2011, pp. 158-207. MR2807831

[Nav18] Andrés Navas, Group actions on 1-manifolds: a list of very concrete open questions, Proceedings of the International Congress of Mathematicians-Rio de Janeiro 2018. Vol. III. Invited lectures, World Sci. Publ., Hackensack, NJ, 2018, pp. 2035-2062. MR3966841

[Ros09] Christian Rosendal, Automatic continuity of group homomorphisms, Bull. Symbolic Logic 15 (2009), no. 2, 184214, DOI 10.2178/bsl/1243948486. MR2535429

[RS07] Christian Rosendal and Sławomir Solecki, Automatic continuity of homomorphisms and fixed points on metric compacta, Israel J. Math. 162 (2007), 349-371, DOI 10.1007/s11856-007-0102-y. MR2365867

[Rub89] Matatyahu Rubin, On the reconstruction of topological spaces from their groups of homeomorphisms, Trans. Amer. Math. Soc. 312 (1989), no. 2, 487-538, DOI 10.2307/2001000. MR988881

[Spa04] R. J. Spatzier, An invitation to rigidity theory, Modern dynamical systems and applications, Cambridge Univ. Press, Cambridge, 2004, pp. 211-231. MR2090772

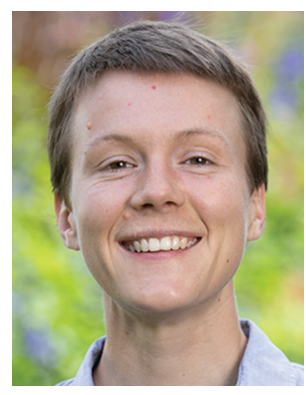

Kathryn Mann

Credits

The opening image is courtesy of ermess via Getty.

All figures are courtesy of the author.

Photo of the author is courtesy of Cornell University/Chris Kitchen. 